\title{
Guía para valoración de lesiones oculares en la práctica forense
}

\section{Guidelines for ocular injuries assessment in forensic medicine}

Con relativa frecuencia, el médico forense tiene que valorar las lesiones y eventuales secuelas oculares de un paciente que ha sido víctima de una agresión o algún tipo de accidente (de tráfico, laboral, deportivo...) que conlleve responsabilidad civil por parte del causante.

El objetivo de esta Guía es resumir los aspectos esenciales que el médico forense o valorador debe tener en cuenta, aportando una información básica y actualizada sobre las técnicas exploratorias y las pruebas complementarias que hoy día se emplean en la práctica oftalmológica asistencial.

El perito médico que debe valorar a un lesionado ocular se enfrenta a una serie de dificultades específicas de las lesiones oftalmológicas:

- Así como en otras ramas de la Medicina Legal (Traumatología, Psiquiatría, Ginecología, Medicina del Trabajo...) no tiene dificultades para manejarse, en la especialidad de Oftalmología surgen dificultades a la hora de interpretar los informes asistenciales, o explorar al paciente, no solo por no disponer del instrumental necesario, sino porque muchas de las técnicas exploratorias oftalmológicas (biomicroscopía, tonometría, oftalmoscopia, gonioscopia...) precisan de un entrenamiento previo que solo tiene el especialista.

- Muchas veces el lesionado presenta un estado anterior (disminución de agudeza o del campo visual, lesiones o degeneraciones retinianas, cataratas...) que actúa como concausa anterior que debemos tener muy en cuenta a la hora de determinar las consecuencias exactas del traumatismo que estamos valorando. Algunas veces el lesionado aportará informes médicos de su función ocular previa a los hechos, pero si no disponemos de esta documentación, el ojo contralateral puede ayudarnos en casos de patología sistémica que ocasione afectación bilateral (retinopatía diabética o hipertensiva, cataratas seniles o metabólicas, degeneración macular asociada a la edad, glaucoma...). En estos casos siempre tendremos en cuenta que la afectación binocular no es simétrica, por lo que ambos ojos no siempre tienen el mismo grado de afectación.

- Otra dificultad es la alta incidencia de simulación que vemos en relación a las secuelas oculares. Así, en el 14\% de los demandantes de una invalidez por causa visual no se evidenció ningún tipo de patología visual ${ }^{1}$, y en el $22 \%$ de los lesionados que reclamaban secuelas oculares teóricamente consecutivas a un accidente de tráfico no encontramos lesiones objetivas que justificaran las molestias referidas por el lesionado, o bien estas no guardaban relación evidente con el accidente ${ }^{2}$. Puede contribuir a esta alta incidencia de simulación el hecho de que la exploración de la agudeza visual es una prueba de tipo subjetivo. Consideramos que, en relación a las secuelas oculares, para dar valor a la sintomatología subjetiva referida por el lesionado debemos poder relacionarla con lesiones y hallazgos exploratorios objetivos que la justifiquen; de lo contrario, tendrá escaso valor desde el punto de vista pericial ${ }^{3}$.

- Finalmente, otro problema importante en estos casos está en relación con las dificultades en el manejo de los baremos. En el apartado de lesiones oculares, algunos de los baremos que habitualmente empleamos son complejos, incompletos o incluso presentan errores o incorrecciones, como comentaremos más adelante, que no facilitan al médico valorador no oftalmólogo la ubicación de muchas secuelas oculares habituales.

La intervención pericial del médico, en estos casos, se desarrolla respecto a cuatro aspectos fundamentales:

- Interpretación y valoración de los informes oftalmológicos asistenciales que constan en el

\author{
JA. Menéndez de Lucas ${ }^{1,2,3}$ \\ V. Molina Seoane ${ }^{2,3}$ \\ F. Luque Mialdea ${ }^{3,4}$ \\ ${ }^{1}$ Médico Forense \\ Especialista en \\ Oftalmología. Clínica \\ Médico Forense. Madrid. \\ ${ }^{2}$ Médico Adjunto. \\ Servicio de Oftalmología. \\ H. U. Montepríncipe. Madrid. \\ ${ }^{3}$ Profesor de Oftalmología. \\ Universidad San Pablo-CEU. \\ Madrid. \\ ${ }^{4}$ Jefe de Servicio de \\ Oftalmología. \\ H. U. Montepríncipe. Madrid.
}

\section{Correspondencia:}

José Antonio Menéndez de Lucas

Sección de Oftalmología.

Clínica Médico Forense de Madrid.

Edificio Juzgados de Instrucción.

Plaza de Castilla núm. 1

28046 Madrid

Email:jamenendezdelucas@yahoo.es

Fecha de recepción:

03.MAR.2015

Fecha de aceptación:

16.MAR.2015 
procedimiento o que son aportados por el lesionado en el momento del reconocimiento.

- Interpretación y valoración de las pruebas complementarias aportadas a las actuaciones.

- Exploración oftalmológica básica con los medios de que habitualmente dispone un forense generalista.

- Valoración de las secuelas oculares en relación al baremo habitualmente empleado en la práctica pericial actual en España: el denominado "baremo de tráfico" (Ley 34/2003).

No pretendemos aquí tratar aspectos concretos de la patología ocular, que claramente exceden el propósito de esta guía, y que el lector puede consultar en uno de los magníficos tratados de oftalmología clínica publicados ${ }^{4}$. Nos centraremos en cuestiones generales que el médico forense debe tener en cuenta en relación a los cuatro puntos anteriores.

Tabla 1. Abreviaturas habitualmente empleadas en oftalmología.

\begin{tabular}{ll} 
Abreviatura & Significado \\
\hline AFG & Angiografía con fluoresceína \\
AO & Ambos ojos \\
AV & Agudeza visual \\
AV CC & Agudeza visual con corrección \\
AV SC & Agudeza visual sin corrección \\
AV CAE & Agudeza visual con agujero estenopeico \\
BMC & Exploración con lámpara de hendidura \\
CV & Campo visual \\
DPA & Defecto pupilar aferente \\
F+ o F- & Tiñe con fluoresceína o no tiñe con \\
FO & fluoresceína \\
LIO (= IOL) & Fondo de ojo \\
MOE & Motilidad ocular extrínseca \\
MOI & Motilidad ocular intrínseca \\
NME & No mejora con estenopeico (la agudeza \\
visual) & \\
OCT & Tomografía de coherencia óptica \\
OD & Ojo derecho \\
OI & Ojo izquierdo \\
PICNR & Pupilas isocóricas y normorreactivas \\
PIO & Presión intraocular \\
PVE & Potenciales visuales evocados \\
TYNDALL + & Inflamación de la cámara anterior (iritis) \\
\hline & \\
\hline &
\end{tabular}

\section{Interpretación de informes oftalmológicos}

La información contenida en los informes asistenciales aportados es esencial para que el médico forense contraste la información que le refiere el lesionado durante la anamnesis.

En el caso concreto de los informes asistenciales oftalmológicos, con frecuencia el perito generalista tiene dificultades para interpretar y a veces "descifrar" la información en ellos contenida 5 . Algunas de estas dificultades se deben al desconocimiento de las abreviaturas que habitualmente se emplean en la práctica clínica oftalmológica actualmente. En la Tabla 1 recogemos algunas de las más habituales.

Describimos a continuación brevemente el contenido de los epígrafes que en general encontramos en un informe clínico oftalmológico, reflejando entre paréntesis el significado de las abreviaturas de uso habitual:

- MC (motivo de consulta) o bien MU (motivo de la urgencia): se describe la sintomatología por la que el paciente consulta o acude al servicio de urgencias.

- Anamnesis: se reflejan los antecedentes del lesionado. Estos pueden ser:

- Antecedentes familiares (AF), en relación a las enfermedades oculares con predisposición genética (glaucoma, retinitis pigmentaria, miopía magna...).

- Antecedentes patológicos generales (AG), como diabetes, hipertensión arterial o hipertiroidismo. También se harán constar las alergias medicamentosas.

- Antecedentes oftalmológicos (AOF), como ametropías, glaucoma, cirugía de cataratas o traumatismos oculares previos.

- Otros antecedentes de interés: como la profesión habitual del paciente, especificando aquellas circunstancias que tengan repercusión sobre la visión. Ya sabemos que la edad predispone a ciertas patologías (presbicia, glaucoma, cataratas, degeneraciones retinianas...) y también haremos referencia a la exposición a agentes tóxicos, así como a tratamientos seguidos (corticoides, cloroquina, antidepresivos...) que producen efectos secundarios que podrían afectar a la función visual.

- Exploración oftalmológica básica: habitualmente consta de las siguientes partes: 
- Inspección ocular: describiendo cualquier alteración morfológica o funcional apreciable a simple vista en los anejos oculares (malformaciones, alteraciones palpebrales o de vías lagrimales).

- Exploración de la musculatura ocular intrínseca (MOI): las pupilas pueden ser isocóricas y normorreactivas (PICNR), o existir un defecto pupilar aferente (DPA) en alguno de los ojos.

- Exploración de la musculatura ocular extrínseca (MOE): puede haber forias o estrabismos, o restricciones de la motilidad ocular extrínseca en cualquiera de las seis posiciones diagnósticas de la mirada, que más adelante describiremos.

- Determinación de la agudeza visual (AV): en cada ojo por separado, para lejos (AVL) o cerca (AVC), ya sea sin corrección (AVsc) o con corrección (Avcc), ya sea en gafa o lentilla. Suele indicarse si mejora con el agujero estenopeico (CAE) o no (NME).

- Examen biomicroscópico (BMC): consiste en el examen de las estructuras del polo anterior (córnea, cámara anterior, iris, cristalino...) mediante la lámpara de hendidura (Figura 1). Es la exploración esencial en oftalmología, ya que permite examinar estas estructuras magnificadas, en visión estereoscópica y determinando la profundidad de las lesiones que asientan en estructuras transparentes como la córnea y el cristalino (Figura 2).

- Tonometría (PIO): es la medición de la presión del humor acuoso mediante un tonómetro de aplanación, previa instilación de una gota de colirio anestésico y con fluoresceína (Fluotest $\left.^{\circledR}\right)$. En condiciones normales suele ser inferior a $21 \mathrm{mmHg}$.

- Oftalmoscopia (FO): consiste en el examen de las estructuras del fondo de ojo (retina, vasos, papila...). En la práctica clínica oftalmológica se emplea habitualmente el oftalmoscopio binocular, que proporciona una imagen estereoscópica e invertida de la retina. Esta exploración suele requerir la instilación previa de un colirio midriático (dilatador pupilar), y permite explorar tanto la retina central como la periférica, así como la cavidad vítrea (Figura 3).

El informe oftalmológico finaliza con el apartado de juicio clínico (JC), donde se recogen los diagnósticos que reflejan los hallazgos patológicos encontrados en el paciente, y el apartado de tratamiento (Tto), en el que se reflejan las prescripciones terapéuticas realizadas, así como la necesidad de revisiones ulteriores.

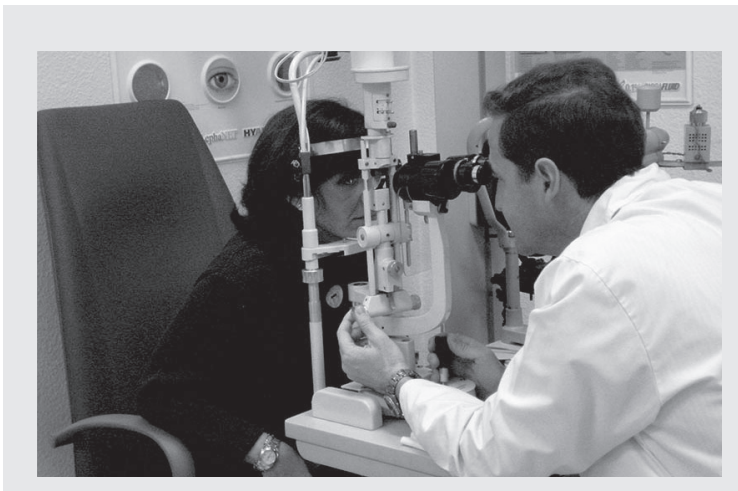

Figura 1.

Exploración con la lámpara de hendidura o biomicroscopía.
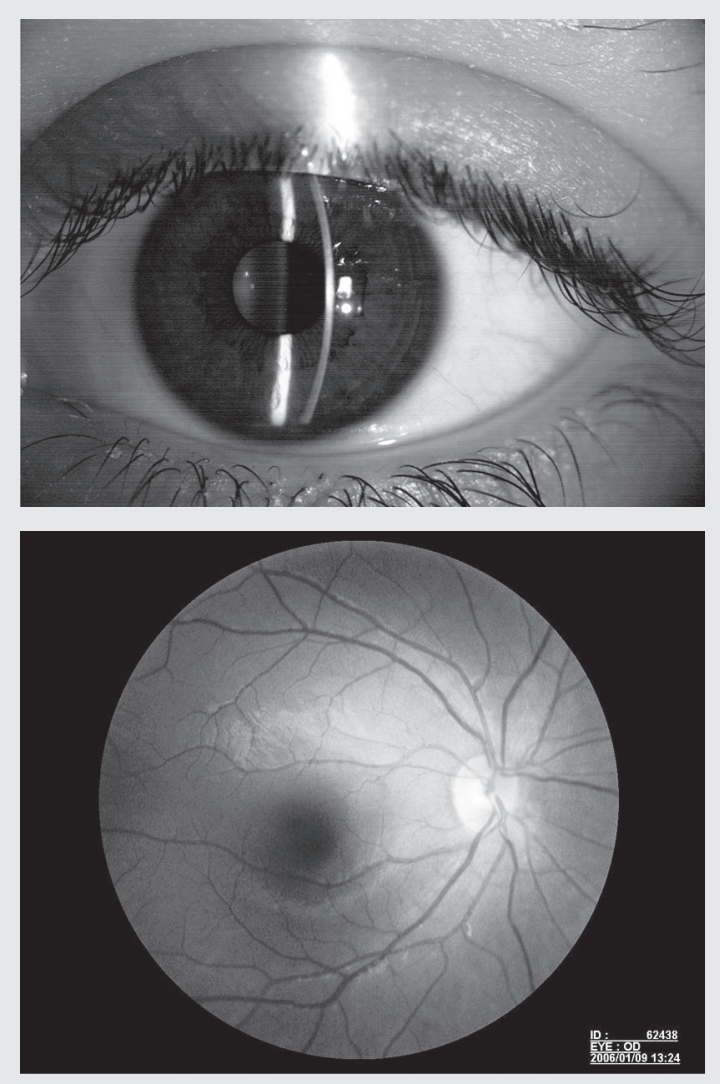

Figura 2.

Imagen del polo anterior del ojo normal con la lámpara de hendidura, donde se aprecia un primer corte del haz luminoso a nivel de la córnea, un segundo en la cara anterior del iris, otro tercero en la cristaloides anterior $y$, finalmente, la luz incide sobre la corteza y el núcleo del cristalino transparente.

\section{Figura 3.}

Imagen de un fondo de ojo normal. Corresponde al $O D$. Puede apreciarse la imagen de la papila en la zona nasal, atravesada por la arteria central de la retina (ACR) que se divide en los vasos temporales superiores e inferiores, $y$ nasales superiores e inferiores. En el centro de la imagen se ve una mancha oscura que corresponde a la mácula, que es la encargada de la visión central.

\section{Interpretación de pruebas complementarias oftalmológicas}

Con frecuencia, el lesionado aporta las pruebas complementarias que le ha realizado su oftalmólogo para tratar de evidenciar determinadas patologías que requieren técnicas exploratorias especiales y a veces de compleja interpretación.

Tiene importancia conocer las indicaciones de este tipo de pruebas y la información que podemos esperar 
Figura 4. Rejilla de Amsler. Puede ser útil para explorar alteraciones de la visión central, pero aplicada al ámbito pericial tiene el inconveniente de ser una prueba de tipo subjetivo (requiere la colaboración del lesionado).

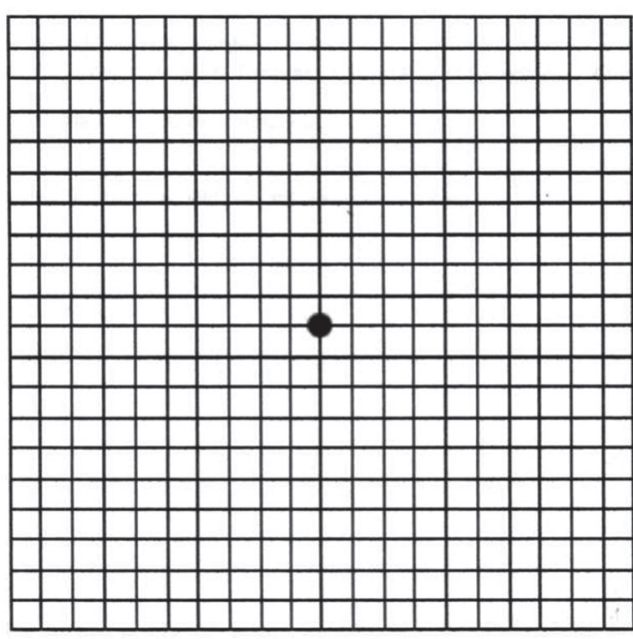

Figura 5.

La OCT macular es esencial dentro del ámbito pericial para tener una constancia documental de las maculopatías postraumáticas.

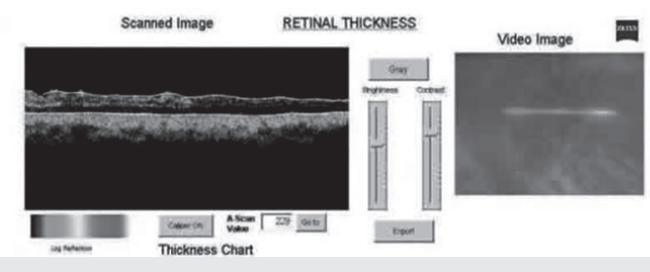

de ellas. Muchas de estas pruebas son esenciales a la hora de tener una constancia documental objetiva que justifique las molestias subjetivas que refiere el lesionado. Entre las pruebas complementarias más frecuentemente solicitadas en oftalmología destacamos:

- Campimetría o perimetría: es la exploración del campo visual. Actualmente se realiza de forma computarizada, y ya no se emplea en la práctica clínica el campímetro clásico de Goldmann. La exploración del campo visual (CV) no solo tiene interés en el seguimiento y el control del curso evolutivo del glaucoma, sino que también es muy útil para detectar alteraciones campimétricas secundarias a patología neuro-oftalmológica postraumática. El campo visual periférico puede explorarse de una forma mucho más sencilla, aunque menos precisa, realizando una campimetría por confrontación, que puede resultar útil para detectar escotomas absolutos que afecten a grandes áreas del campo visual (hemianopsias o cuadrantanopsias). Por otro lado, el campo visual central puede explorarse de manera rápida y sencilla empleando la rejilla de Amsler (Figura 4).
- Gonioscopia: consiste en visualizar el ángulo de la cámara anterior mediante una lente especial (lente de Goldmann de tres espejos), que se coloca en contacto con la córnea aplicando un gel lubrificante y permite ver las estructuras de esta zona y detectar una posible recesión angular, así como cuantificar su amplitud.

- Técnicas exploratorias corneales: algunas de ellas tienen interés pericial, principalmente en relación con las denuncias por presunta mala praxis médica en intervenciones de cirugía refractiva con láser. Así, destacan la topografía corneal, que proporciona un mapa de alturas corneales en diferentes colores, y la paquimetría, que consiste en medir el espesor corneal.

- Biomicroscopía endotelial: permite fotografiar y realizar un recuento celular de esta capa de la córnea, con vistas a indicar o no intervenciones de cirugía de cataratas mediante facoemulsificación.

- Retinografía: consiste en fotografiar mediante cámaras especiales la retina, para registrar los detalles del fondo de ojo y así poder hacer comparaciones futuras. Son imágenes en color y de gran calidad, que pueden almacenarse, ya que en la actualidad se emplean imágenes digitalizadas (Figura 3).

- Angiografía con fluoresceína (AFG): se realizan fotografías seriadas del fondo de ojo, habitualmente en blanco y negro, para analizar la circulación de un colorante (fluoresceína) que se inyecta en la vena cubital y alcanza el ojo a través de la arteria central de la retina (tiempo arterial), difunde a los capilares y se elimina del ojo por las venas retinianas (tiempo venoso). Esta prueba se emplea para estudiar alteraciones circulatorias retinianas y afecciones maculares, y para establecer las indicaciones de tratamientos mediante fotocoagulación con láser argón.

- Tomografía de coherencia óptica (OCT): se emplea en clínica habitualmente para valorar la papila óptica y la mácula. En la actualidad tiene un especial interés pericial la OCT macular (Figura 5), para tener una constancia documental en los casos de maculopatías postraumáticas, que pueden ser secuelas de los traumatismos oculares que lesionan el polo posterior del ojo.

- Pruebas electrofisiológicas oculares: tienen especial interés médico-legal, en relación con la simulación, los potenciales visuales evocados (PVE). Esta prueba consiste en recoger la respuesta eléctrica a nivel de la corteza visual, sometiendo al paciente a un estímulo visual 
estandarizado. Cuando el trazado es correcto, indica indemnidad de la vía óptica. El trazado de los PVE puede alterarse incrementando la latencia o disminuyendo la amplitud de sus ondas (principalmente de la onda P100). Dudaremos, en principio, de una aparente ceguera que nos aporte unos PVE normales (no alterados) en ese ojo, aunque debemos tener muy en cuenta que unos PVEP (con estímulo pattern) alterados en un ojo no implican necesariamente una alteración de la vía óptica en ese sujeto, ya que podemos encontrar respuestas "anormales" producidas voluntariamente (simuladas) en sujetos normales entrenados ${ }^{6}$.

- Ecografía ocular y orbitaria: la ultrasonografía está especialmente indicada cuando existe una turbidez de medios que impide conocer el estado de la retina, o para detectar cuerpos extraños intraoculares. Es en particular útil en traumatismos en los que no puede descartarse la existencia de un desprendimiento de retina por opacidades de los medios transparentes del ojo.

- Pruebas de imagen: la radiología simple (Rx) y la tomografía computarizada (TC) son en especial útiles para valorar alteraciones oculares y orbitarias en relación con los traumatismos, como fracturas craneales u orbitarias, cuerpos extraños intraoculares o intraorbitarios de naturaleza metálica. La resonancia magnética (RM) debe evitarse si sospechamos la existencia de un cuerpo extraño intraocular (CEIO) metálico o buscamos lesiones óseas, pero puede ser muy útil para valorar lesiones de partes blandas (compresiones del nervio óptico, atrapamiento de los músculos extraoculares, edemas o hemorragias postraumáticas).

\section{Exploración oftalmológica básica que puede realizar el médico forense}

Cinco sencillas exploraciones oftalmológicas básicas nos van a permitir resolver la mayoría de los problemas que puedan surgir con un lesionado que presente alguna patología ocular. El material exploratorio necesario para realizarlas está al alcance de cualquiera y debería estar presente en todo despacho donde desarrolle su actividad el médico forense. En principio nos bastaría con un póster de optotipos colocado a la distancia indicada por el fabricante, un agujero estenopeico (Figura 6) que eliminará los defectos de refracción del paciente para obtener la mejor agudeza visual corregida (que es la que nos in-

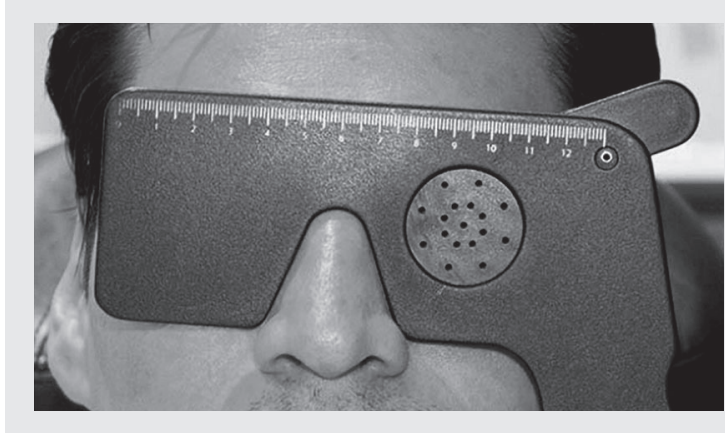

Figura 6.

El agujero estenopeico permite explorar la mejor agudeza visual corregida del lesionado, es decir, nos indica la agudeza visual que podría alcanzar si corrigiéramos los posible defectos de refracción (miopía, hiperme tropía o astigmatismo) que pueda tener. teresa a efectos periciales), una pequeña linterna para explorar la motilidad ocular intrínseca y extrínseca, y un oftalmoscopio directo para ver el fondo de ojo.

Con este material podremos realizar las cinco siguientes técnicas exploratorias oftalmológicas básicas, que en muchos casos van a permitir tener un conocimiento directo de la patología que sufre el lesionado y no limitarnos a hacer una mera transcripción de los informes oftalmológicos aportados a las actuaciones:

\section{Determinación de la agudeza visual}

Es la prueba más importante de la función visual, y la más utilizada. Nos da una idea cuantitativa del grado de visión central que tiene el sujeto, que es la que se emplea al mirar un objeto lejano o al leer. Podemos determinar la agudeza visual para lejos o para cerca, y estas a su vez pueden medirse con o sin la corrección. A efectos periciales, la que más nos interesa es la visión de lejos y con corrección, ya que la mayoría de las secuelas oculares no afectan a la refracción del lesionado, y la agudeza visual de cerca habitualmente es equiparable a la de lejos una vez corregida la eventual presbicia que pueda sufrir el lesionado. La agudeza visual es la capacidad para percibir como separados dos puntos que están muy próximos, y dadas las características anatómicas de la retina, estos deben estar separados más de 1 minuto de arco. Podemos expresar la AV como un quebrado en el cual el numerador es la distancia a la que ve el paciente un optotipo (por ejemplo, el de Snellen) y el denominador es la distancia a la que debería verlo si tuviera una agudeza normal o de la unidad, que en este caso sería 6 metros (20 pies). Así, por ejemplo, una agudeza visual de 1/4 quiere decir que el sujeto tiene que acercarse a 1 metro de distancia para ver un letrero (la matrícula de un coche, por ejemplo) que un sujeto con visión unidad sería capaz de leer a 4 metros; o una visión de 1/10 
quiere decir que el sujeto tiene que acercarse a 1 metro para leer lo que un sujeto normal vería a 10 metros. También podemos expresar la AV como el número decimal resultante de la fracción, y así 1/4 sería 0,25, o 1/10 sería 0,10 (Tabla 2). Cuando la visión es muy baja decimos que "cuenta dedos a 1 metro", o "solo es capaz de ver el bulto de la mano", o "tan solo percibe la luz". La técnica para determinar la agudeza visual es sencilla. Comenzaremos tapando el ojo izquierdo (OI) del paciente para explorarle el ojo derecho (OD), con sus gafas puestas en el caso de que las use. Debe estar situado a la distancia adecuada de los optotipos, generalmente a 5 metros. Anotamos la última fila que es capaz de leer correctamente con ese OD, y luego, sin destapar el Ol, le pondremos el agujero estenopeico frente al OD para ver si mejora y es capaz de leer alguna fila más. Si el lesionado es capaz de leer hasta la fila de 0,6 y al ponerle el estenopeico ve también las filas de 0,7 y 0,8, pero no ve la fila de 0,9 , expresaremos la agudeza visual como: AV OD lejos (cc) = 0,6 CAE 0,8; es decir, la agudeza visual corregida, de lejos, en el ojo derecho, es de 0,6, y con agujero estenopeico mejora a 0,8. A continuación taparemos el OD para explorar la visión del OI.

\section{Campimetría por confrontación}

Consiste en explorar el campo visual del paciente comparándolo con el del explorador, que consideramos normal. Nos sentamos frente al lesionado, aproximadamente a 1 metro de distancia. Para explorarle el OD debemos taparle el izquierdo y pedirle que mire a nuestro OI. Vigilamos que no desvíe la mirada durante la exploración. Guiñamos nuestro OD y exploramos los cuatro cuadrantes del campo visual (superior, inferior, temporal y nasal), introduciendo un bolígrafo perpendicularmente desde la zona más periférica del cuadrante explorado, y comparamos cuándo empieza a verlo "de reojo" y cuándo empezamos a verlo nosotros. El campo visual normal tiene una amplitud de unos $60^{\circ}$ en los sectores superior y nasal, unos $70^{\circ}$ en el sector inferior y $90^{\circ}$ en el sector temporal. Con este tipo de campimetría solamente podremos detectar escotomas absolutos groseros, y no escotomas relativos como los que se producen en los estadios iniciales del glaucoma, pero estos defectos campimétricos absolutos son los que habitualmente nos interesan en la práctica médico-legal y forense. Podemos así contrastar nuestra exploración con las campimetrías computarizadas que habitualmente aporta el lesionado, ya que los escotomas pueden haber evolucionado, ya sea hacia la mejoría o hacia el agravamiento, con lo que es posible que la situación actual no se corresponda exactamente con la reflejada en los informes.

\section{Exploración de la motilidad ocular intrínseca (MOI)}

Es relativamente fácil explorar la $\mathrm{MOI}$ empleando una pequeña linterna de bolsillo, en una habitación que esté en penumbra. Exploramos el reflejo fotomotor en ambos ojos: cuando iluminamos uno de los ojos se produce una contracción de la pupila o miosis en ese ojo, que es la respuesta directa, y también se produce una contracción simultánea en el ojo contralateral, que es el reflejo indirecto o consensual. Tiene especial interés médico-forense detectar un defecto pupilar aferente (DPA): el ojo que lo sufre no tiene reflejo fotomotor directo, lo cual nos indica que no percibe la luz, es decir, que está amaurótico o ciego. En un lesionado que refiere ceguera de un ojo en el que no objetivamos un DPA, debemos plantearnos que se trate de un simulador.

\section{Exploración de la motilidad ocular extrínseca (MOE)}

Nos sentamos a 1 metro de distancia frente al lesionado, que en este caso está con los dos ojos destapados. Le pedimos que mire con ambos ojos a nuestro bolígrafo, aunque lo vea doble. Debe mantener la mirada en el bolígrafo, que vamos colocando en posición primaria de la mirada (ppm) y en las seis posiciones diagnósticas: dextroelevación, dextroversión, dextrodepresión, levoelevación, levoversión y levodepresión. Estas posiciones se denominan así porque en cada una de ellas actúa predominantemente un músculo extraocular de un ojo y otro músculo concreto del ojo contralateral. Una restricción de la movilidad en cada una de las posiciones diagnósticas se corresponderá con una parálisis de un determinado músculo, en función del lado que esté afectado. Se exploran las versiones o movimientos de ambos ojos de manera sincrónica y simétrica en la misma dirección.

\section{Examen del fondo de ojo mediante un oftalmoscopio directo}

Pedimos al lesionado que enfoque un objeto a lo lejos, mientras observamos a través del oftalmoscopio directo las estructuras del interior del globo ocular. Con esta técnica nunca llegaremos a ver la retina con estereopsis y con la amplitud con la que la ve el especialista, que habitualmente emplea un oftalmoscopio indirecto binocular bajo midriasis, pero puede servirnos para visualizar en muchos casos las estructuras del polo posterior (papila y mácula). 


\section{Valoración de las secuelas oculares con el baremo de la Ley 34/2003}

Este baremo es, sin lugar a dudas, el más empleado en España para la valoración de secuelas, ya que se utiliza para fijar las indemnizaciones derivadas de todo tipo de accidentes que generen responsabilidad civil (laborales, deportivos, de caza...), teniendo además carácter vinculante en el caso de los accidentes de tráfico. También se emplea en la Jurisdicción Penal para fijar las indemnizaciones por responsabilidad civil derivada de la criminal, de conformidad con una circular de la Fiscalía. Incluso se utiliza actualmente, con carácter orientativo, para fijar las indemnizaciones por responsabilidad profesional médica, al no existir aún un baremo específico para estos casos. Es, por lo tanto, el baremo más importante y el más utilizado hoy en día para la valoración del daño corporal.

Lamentablemente, en nuestra opinión, en el apartado de valoración de las secuelas oculares existe una serie de errores o carencias. A los pocos meses de aprobarse publicamos una crítica constructiva ${ }^{7}$ en la que explicábamos que en dicho baremo se introducen elementos que carecen de fundamento científico, como pretender valorar un leucoma corneal por el déficit campimétrico que ocasiona, en lugar de hacerlo por el déficit de agudeza visual, o proponer añadir una serie de puntos adicionales en las lesiones postraumáticas del iris "en caso de trastorno de la acomodación", cuando, como es sabido, la acomodación se realiza mediante el músculo ciliar y no mediante el iris. Tampoco aclaraba definitivamente que la exploración de la agudeza visual que tiene trascendencia a la hora de valorar las secuelas postraumáticas es la de lejos y con corrección, como hemos explicado antes al hablar de la exploración de la agudeza visual. Esta es una cuestión que lleva años planteando discusiones innecesarias y errores que serían perfectamente evitables aclarándolo. Algo parecido ocurre con la forma de expresar la agudeza visual del lesionado, que como ya hemos dicho debería ser en forma decimal, porque es la que actualmente se emplea en la práctica clínica. Este baremo sigue expresando la agudeza visual en la Tabla $A$ en forma de quebrado, que hoy resulta obsoleta, y complica de manera innecesaria la valoración en la práctica. Incluye un factor corrector de la disminución de la pérdida de agudeza visual en aquellos casos en los que el ojo lesionado sufriera un déficit visual previo (estado anterior). Sin embargo, debemos ser coherentes e introducir un factor corrector de agravamiento en aquellos casos en los que la lesión se produce en un "ojo único" o con muy baja visión en el ojo contralateral. No se hace referencia a este factor de agravamiento en la actual Tabla IV.

Además, consideramos que es un baremo incompleto, ya que no recoge secuelas típicamente postraumáticas, como son una recesión angular que puede dar lugar a un glaucoma postraumático, o el desprendimiento del vítreo posterior, que es la secuela ocular más frecuente en los accidentes de tráfico ${ }^{2}$. Tampoco figuran la erosión corneal recidivante ni el menoscabo sufrido en un ojo que ha sido vitrectomizado o fotocoagulado como consecuencia de un desgarro o un desprendimiento de retina postraumático, y que puede no haber reducido su agudeza visual, o la catarata que suele desarrollar un ojo en el que se ha realizado una vitrectomía para tratar un desprendimiento de retina postraumático. Evidentemente, un baremo no puede recoger todas las lesiones posibles, pero sí debería hacerlo con las típicas postraumáticas para evitar polémicas innecesarias.

Todas estas deficiencias que, en nuestra opinión, presenta el baremo, tuvimos la ocasión de subsanarlas cuando en el año 2007 fuimos invitados a participar en el comité científico que elaboró el Baremo del Instituto de Medicina Legal y Ciencias Forenses de

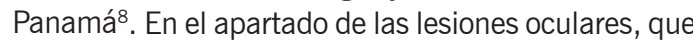
incluimos en la Tabla 3, eliminamos los errores antes descritos, introdujimos aquellas secuelas típicamente postraumáticas, aclaramos de manera explícita que la agudeza visual debe valorarse con corrección, y eliminamos la Tabla B (visión de cerca) por considerarla innecesaria. Simplificamos la valoración de la diplopía atendiendo a su repercusión funcional, mayor cuando afecta a la mirada al frente o hacia abajo. También añadimos un factor de agravamiento en las secuelas que afecten a un "ojo único". En la Tabla A expresamos la agudeza visual de lejos en la forma en que habitualmente se hace en la práctica oftalmológica en Panamá por la influencia norteamericana (números quebrados con numerador 20, por ejemplo 20/20, 20/40, etc.). En lugar de puntos, en este baremo las cantidades expresan porcentajes y se modifican ligeramente algunas de las normas de aplicación, pero en esencia es el de la Ley 34/2003 y actualmente se emplea en el Instituto de Medicina Legal y Ciencias Forenses de Panamá con buenos resultados.

En el año 2011, el comité técnico de la Unión Española de Entidades Aseguradoras y Reaseguradoras (UNESPA) solicitó la colaboración de los médicos forenses a través de los Institutos de Medicina Legal y Clínicas Médico Forenses de toda España, por lo que tuvimos ocasión de hacerle partícipe de nuestras sugerencias y propuestas de modificación. La 
Tabla 2

Equivalencia de diferentes formas de expresar la agudeza visual.

\begin{tabular}{lcccc}
\hline Snellen & Quebrado & Porcentaje jurídico & Decimal & Eficiencia \\
\hline $6 / 6$ & $10 / 10$ & $100 \%$ & 1 & $100 \%$ \\
$6 / 6,7$ & $9 / 10$ & $90 \%$ & 0,9 & $98 \%$ \\
$6 / 7,5$ & $8 / 10$ & $80 \%$ & 0,8 & $96 \%$ \\
$6 / 8,6$ & $7 / 10$ & $70 \%$ & 0,7 & $92 \%$ \\
$6 / 10$ & $6 / 10$ & $60 \%$ & 0,6 & $84 \%$ \\
$6 / 12$ & $5 / 10$ & $50 \%$ & 0,5 & $78 \%$ \\
$6 / 15$ & $4 / 10$ & $40 \%$ & 0,4 & $52 \%$ \\
$6 / 20$ & $3 / 10$ & $30 \%$ & 0,3 & $40 \%$ \\
$6 / 30$ & $2 / 10$ & $20 \%$ & 0,2 & $25 \%$ \\
$6 / 60$ & $1 / 10$ & $10 \%$ & 0,1 & $15 \%$ \\
$6 / 90$ & $1 / 20$ & $5 \%$ & 0,05 & $10 \%$ \\
Bultos & Bultos & Bultos & Bultos & $5 \%$ \\
Luz & Luz & Luz & Luz & $2 \%$ \\
Ceguera & Ceguera & Ceguera & Ceguera & $0 \%$ \\
\hline
\end{tabular}

Tabla 3. Propuesta de modificaciones al baremo de la Ley $34 / 2003$.

\section{Sistema ocular}

Globo ocular:

Ablación de un globo ocular

Ablación de ambos globos oculares

Polo anterior:

Leucoma; valorar según pérdida de agudeza visual, añadiendo 2 puntos si ocasiona diplopía monocular

Erosión corneal recidivante o queratalgia recidivante

Alteraciones postraumáticas de iris (discorias, iridodiálisis, midriasis postraumáticas), valorar en función del grado de deslumbramiento

Recesión angular que pudiera ocasionar un glaucoma postraumático en el futuro:

Entre $90^{\circ}$ y $270^{\circ}$

Mayor de $270^{\circ}$

Glaucoma postraumático

Cristalino:

Catarata postraumática sin indicación quirúrgica; valores según agudeza visual y añadir 5 puntos

Afaquia unilateral tras fracaso quirúrgico; valorar según trastorno de la agudeza visual y añadir 5 puntos

Colocación de lente intraocular

Rotura zonular postraumática que ocasione facodonesis

Anejos oculares:

Entropión, tripiasis, ectropión, cicatrices viciosas, lagoftalmos

Ptosis palpebral no operable (se valora el déficit funcional al que se añadirá el perjuicio estético):

Unilateral

Bilateral 
Epífora y ojo seco postraumático:

Unilateral $1-6$

6-12

Manifestaciones hiperestésicas o hipoestésicas periorbitarias

$1-5$

Campo visual:

Visión periférica:

Hemianopsias:

Homónimas

Heterónimas:

Nasal

Temporal

Cuadrantanopsias:

Nasal inferior

Nasal superior

3-8

Temporal inferior

3-8

Temporal superior

2-7

Escotomas yuxtacentrales

Visión central: escotoma central

Polo posterior:

Metamorfopsias por maculopatía postraumática o agujero macular evidenciados mediante pruebas de imagen (AFG, OCT). Además de la puntuación que le corresponda por la reducción de agudeza visual

Desprendimiento del vítreo posterior postraumático

3

Roturas coroideas, ojo vitrectomizado o que ha precisado tratamiento fotocoagulador preventivo de desprendimiento de retina o colocación de un cerclaje (añadir al valor correspondiente por pérdida de agudeza visual)

Diplopía binocular postraumática no solucionable quirúrgicamente, ni con prismas:

En posiciones extremas de la mirada

En el campo lateral o superior de la mirada

En la parte inferior del campo visual (afectando a la lectura y la deambulación)

En posición primaria de la mirada (al mirar al frente), obligando a ocluir un ojo

Diplopía binocular compensable mediante corrección prismática

5

Agudeza visual:

Déficit de la agudeza visual (consultar Tabla A). Se valora siempre la AV en visión de lejos y con corrección. La visión de cerca siempre es buena si lo es la de lejos o puede solucionarse con tratamiento o con corrección

Pérdida de visión de un ojo

Nota: si el ojo afectado por el traumatismo tenía anteriormente algún déficit visual, la tasa de agravación será la diferencia entre el déficit actual y el existente (factor corrector de disminución de la secuela). Por el contrario, en aquellos casos en los que la lesión se produce en un "ojo único" o con baja visión en el contralateral, habrá que incrementar la puntuación mediante un factor corrector de agravamiento de la secuela

\section{6}

5


mayoría de estas propuestas han sido incluidas en el Proyecto de Ley de modificación del Baremo, que ha sido aprobado en el Consejo de Ministros de 10 de abril de 2015, y está pendiente de tramitación en las Cortes Generales, con previsible entrada en vigor el 1 de enero de 2016. Lamentamos que haya tenido que pasar más de una década desde que publicamos nuestra "crítica constructiva"7 en el año 2004.

Los autores declaran no tener ningún conflicto de intereses.

\section{Bibliografía}

1. García Carcellé VA. La valoración médico-legal en oftalmología. Madrid: Allergan Therapeutic; 1991. p. 13-6.

2. Menéndez de Lucas JA, Pera F, Morcillo R. Valoración de lesiones producidas en los accidentes de tráfico. Cuad Med Forense. 2008;14(51):25-33.

3. Menéndez de Lucas JA, Merayo Llovés J, Fernández Vega L. Valoración del daño corporal en oftalmología: interpretación de informes oftalmológicos y exploración oftalmológica básica en la práctica pericial. Análisis de los principales baremos. En: Delgado Bueno S, editor. Tratado de medicina legal y ciencias forenses. Barcelona: Bosch; 2012. II, p. 1291-313.

4. Kanski JJ. Oftalmología clínica. $6^{a}$ ed. Barcelona: Elsevier España; 2009.

5. Moreno Cantero F, Fagúndez Vargas MA. Traumatismos oculares: aspectos médico-legales. Cuad Med Forense. 2002;(29):5-19. (Consultado el 25 de enero de 2008.) Disponible en: http:// scielo. isciii.es/

6. Menéndez de Lucas JA, Luque Mialdea F, Molina Seoane V. ¿Debemos considerar los potenciales visuales evocados una prueba objetiva? A propósito de un caso. Rev Esp Med Legal. 2011;37(4):16972 .

7. Menéndez JA. Crítica constructiva a las recientes modificaciones del baremo para la valoración de las secuelas de los accidentes de tráfico. Studium Ophthalmologicum. 2004;1:49-51.

8. Menéndez de Lucas JA, Donat Laporta E, Varela Petrucelli LV, Pérez Mendez A, Garcia Alvarado S. Baremo del Instituto de Medicina Legal y Ciencias Forenses de Panamá. Normas de aplicación. Jornada de exposición y validación del baremo organizada por el Ministerio Público, el IML y el fondo mixto de cooperación Hispano-Panameño, en el Hotel Riande Continental, el 23 de marzo de 2007. 